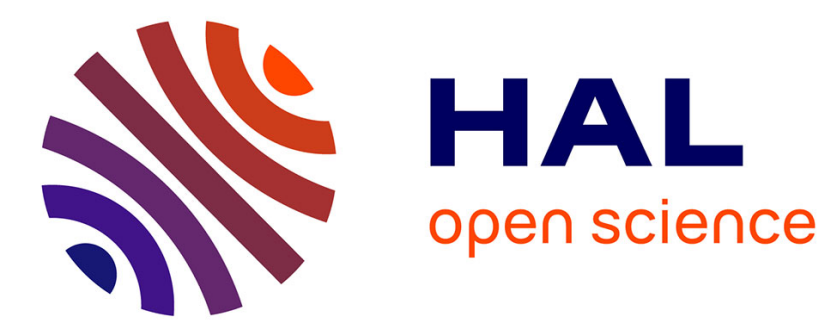

\title{
3D Reconstruction of Blood Vessels
}

Ali Al Moussawi, Cedric Galusinski, Christian Nguyen

\section{To cite this version:}

Ali Al Moussawi, Cedric Galusinski, Christian Nguyen. 3D Reconstruction of Blood Vessels. Engineering with computers, 2014, pp.1-16. 10.1007/s00366-014-0398-3 . hal-01002127

HAL Id: hal-01002127

https://hal-univ-tln.archives-ouvertes.fr/hal-01002127

Submitted on 5 Jun 2014

HAL is a multi-disciplinary open access archive for the deposit and dissemination of scientific research documents, whether they are published or not. The documents may come from teaching and research institutions in France or abroad, or from public or private research centers.
L'archive ouverte pluridisciplinaire HAL, est destinée au dépôt et à la diffusion de documents scientifiques de niveau recherche, publiés ou non, émanant des établissements d'enseignement et de recherche français ou étrangers, des laboratoires publics ou privés. 


\title{
$3 D$ Reconstruction of Blood Vessels
}

\author{
Al Moussawi Ali Galusinski Cedric Nguyen Christian
}

June 5,2014

\begin{abstract}
The aim of this paper is to achieve the $3 D$ reconstruction of blood vessels from a limited number of $2 D$ transversal cuts obtained from scanners. This is motivated by the fact that data can be missing. The difficulty of this work is to connect the blood vessels between some widely spaced cuts in order to produce the graph corresponding to the network of vessels. We identify the vessels on each transversal cut as a mass to be transported along a graph which allows to determine the bifurcation points of vessels. Specifically, we are interested in mass transfer problem Monge-Kantorovich [10] to model an optimized graph associated to the network of vessels. At this stage, we are able to reconstruct a $3 D$ level set function by using the $2 D$ level set functions given by the transversal cuts and the graph information. When the whole scanners data are available, a global reconstruction is proposed in a simple manner, without using the mass transfer problem.
\end{abstract}

\section{Introduction}

$3 D$ reconstruction of blood vessels has been widely developed in recent years in the field of medical research to allow the practitioners to establish correct diagnosis. Several studies describe different numerical approaches to reconstruct a digital modeling of blood vessels closest to reality by using medical imaging [33] which combines the means of acquisition and retrieval of the human body from different physical phenomena such as scanners, intravascular ultrasound (IVUS) and angiographies system.

The aim of this work is to reconstruct $3 D$ blood vessels from medical images even if data are missing. These data are collected from scanner images and correspond to $2 D$ transversal cuts. Our algorithm can be applied indifferently to a reduced number of transversal cuts or to full data. The difficulty and the novelty of this article is to construct an optimized graph associated to the network of vessels and the $3 D$ geometry between two possible widely spaced successive cuts. The reconstructed geometry represents does not include aberrations due to image inaccuracies thanks to the chosen reconstruction technique. Then it is possible to use this reconstructed geometry in a fluid mechanic solver in order to compute flows.

The medical imaging devices are diverse and data imaging are then various. The techniques to achieve $3 D$ reconstruction of blood vessels are briefly described hereafter. The $3 D$ reconstruction is made from medical imaging data such that the angiographic images, the echographic images (IVUS) and the tomographic images (CT-scan). 
Angiographic images are obtained by X-rays to provide longitudinal cuts associated to the vessels. These images can provide information about the thickness of vessels by removing regions of the image that are not relevant, such as the bones and the soft tissues. Methods of $3 D$ reconstruction of blood vessels from the angiographic images are numerous [31],[14],[8], in which the problems encountered by the use of a limited number of projections solved by the introduction of strong geometric assumptions on the blood vessels. Moreover, data provided by the angiographic technique can be damafged by geometric distortions, structural noise and the inhomogeneous of the vessel segment. The obtained results from this procedure are generally imprecise and particularly over-regularized [24].

The echographic images are produced by intravascular ultrasound (IVUS) to provide transversal cuts associated to the vessels. In this case, the $3 D$ reconstruction is limited by the uncertainty on the orientation of the catheter, the non perpendicularity of the ultrasonic radiation on the wall structures and the offset positioning of the catheter [30],[16],[7]. Moreover, The $3 D$ reconstruction by vertical stacking of the images introduce substantial geometric error in vessels [15],[23], because they do not take into consideration the curvature of this vessels which generate a volumetric variation.

To take into consideration the winding in $3 D$ reconstructed of blood vessels from the echographic data, several approaches are presented in [25],[28],[12], using the monoplanes or the biplanes angiographic systems to position the (IVUS) catheter pullback path. The echographic images are aligned along this path. In [6], the method of $3 D$ reconstruction using a biplane angiography system requires a continuous record of the (IVUS) catheter pullback in this system and the use of a calibration cube. Another approach [26],[27] has been developed where a calibration is made and the orientation throughout pullback is estimated using a $3 D$ Fourier function. This method requires a constant velocity of the (IVUS) transducer during a pullback. Methods using biplane angiography systems provide several disadvantages, in particular the complexity of the acquisition process. These systems are not widely available in clinical settings and they produce more radiation on patients. Some use monoplane angiography systems and takes images with different viewing angles, simulating the use of a biplane system. However, this approach complicates the acquisition process, generates additional radiations on the patient and distorting the results if the latter moves during acquisition [17].

The other approach proposed in [25] leads to use one angiographic image. It induces an ambiguity about the depth of the points in the $3 D$ trajectory since a single view is not sufficient. The length of the $3 D$ transducer and its $2 D$ projection are used as a priori information to determine the depth of the points along the trajectory of the transducer. However, the method developed is not robust because the transducer is small and its projection can do sometimes one or two pixels in the angiographic image without leaving a great margin of error for its position in the image. In [12] a new method based on a single-plane angiography system is proposed to construct the $3 D$ (IVUS) transducer tracking. This new method uses the displacement velocity of the transducer as a priori information, rendering such method more robust than the one presented previously in [25]. The $3 D$ velocity of the transducer is constant and known during the pullback process. It is therefore possible to make a link between this $3 D$ velocity and the $2 D$ velocity viewed in the angiographic images to find the $3 D$ positions of the transducer during its pullback. 
The tomography is a technique which is widely used in medical imaging. This technique allows to reconstruct the $3 D$ object from a series of measurements performed by a slice deported outside of the object. We mention the CT-scan which allow to measure the X-ray absorption in the tissues and to reconstruct the $2 D$ or $3 D$ images of anatomical structures by computer processing [1]. If the tomographic data are the projections of the organ studied, then the tomographic reconstruction will be done by solving an inverse problem (for more information about the inverse problems in medical imaging [3]). If the data are the $2 D$ transversal cuts of the organ, these cuts can then be superimposed to each other and the $3 D$ tomographic reconstruction of the studied organ is simply done by stacking. Finally, the reconstruction operates on complete 3D data, restricted by projection. In our approach, we use a limited number of transversal cuts obtained from scanners to reconstruct the blood vessels. The novelty in this article is to construct the paths connecting centers of vessel contours between the $2 D$ transversal cuts obtained by scanners, from an algorithm based on the mass transfer problem and defining the optimal graph corresponding to the core of vessels. Unlike methods used in [25],[28],[12], we don't use longitudinal data obtained by the angiographic system. Unlike conventional methods based on inverse methods on CT-scan [3], we can reconstruct $3 \mathrm{D}$ vessels even if large $3 \mathrm{D}$ data are missing.

This present work is performed in three steps:

- a $2 D$ imagery process to analyse a $2 D$ transversal cut in order to identify vessels,

- a graph construction from an algorithm based on the mass transfer problem,

- the $3 D$ reconstruction from the graph and the images.

The first step of the work is discussed in section 1 and is based on segmentation tools in order to extract vessel contours. A human intervention is necessary to distinguish the vessel contours from the other contours. The second step deals with the graph construction which is developped in section 2. The construction of such a graph is based on a mass transfer problem called Monge-Kantorovich problem [10], by optimizing the cost of the transport of blood along the net of vessels. The mass of transport associated to blood flow, is evaluated with the surface of vessels on cuts.

The $3 D$ reconstruction, detailed in section 3 , is achieved by the definition of a level set function. Level set functions are first defined on $2 D$ cut in section 1 and then interpolated by using the graph information. The validation of results is performed in section 4 on transversal cuts of the abdominal aorta and the femoral artery. Section 5 deals with the way of enriching the initial reconstruction by new cuts without human intervention in image processing by exploiting the initial graph. When full scanners data are available, another approach is investigated in section 6 . We also show how to use tools developed in section 5 without knowing the graph of the geometry. In this case, starting from a human intervention on the first cut, the full geometry is reconstructed.

\section{Recovery of $2 D$ level set data from the medical imaging}

The aim of this section is to extract, from a transversal cut, the vessel contours. By a human intervention, vessels can be identified on the $2 D$ image. We then propose to construct the $2 D$ level set signed distance [11] function whose 0-level corresponds to the vessel boundary. This function 
is negative inside the vessels (the region identified during the human intervention) and positive outside. We explain hereafter how to construct such a function.

\subsection{Extraction of the $2 D$ contours of vessels}

From medical images, like angiographies or dopplers, which are degraded by construction, we have to extract the connected regions of the vessels. These regions are identified by a binary code, for example the outer regions are coded 1 (white color) and the inner regions are coded 0 (black color).
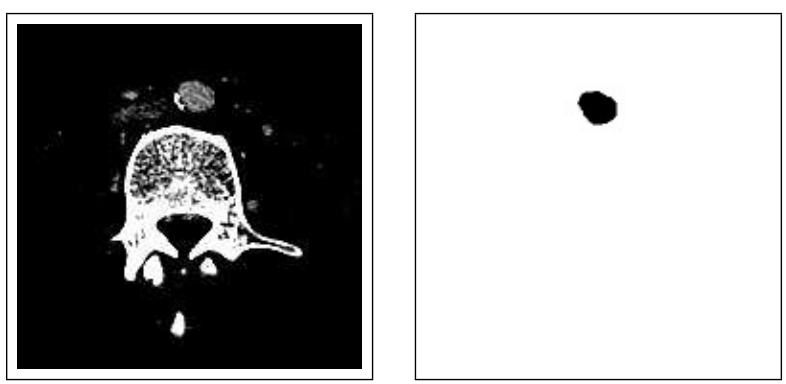

Figure 1: Original medical image on the left, blood vessel extracted on the right

First of all, we use the free software VRRender allowing to render the $3 D$ medical images in DICOM format (itk, ircad, vtk/gdcm). Volume rendering (VR) is a well known visualization method for the $3 D$ visualization of medical images based on transparency and coloration of a set of voxels, each voxel having a grey level that represents a physical property of the tissue (absorption of $\mathrm{X}$ ray in case of computerized tomography (CT) for instance). For medical use, the current software includes several tools like selection of the slice, multi-planar rendering (axial, frontal or sagittal views), improving the visualized grey-level window and pre-computed CT-scan transfer functions allowing to visualize bones, kidneys, liver, lungs, muscles, skin and vessels. Thanks to this, we can save the pictures of the areas of interest.

As most medical images, they lack contrast and have a strong noise which may interfere with obtaining a clean geometry corresponding to a proper image interpretation. To solve these defects, we combine well-known image enhancement algorithms, ranging from simple local or global contrast increasing to linear or non linear filter functions in order to reduce parasitic noises. All of these tools are designed to ease the work of the next step in the extraction of connected regions. To extract the contours most of region-based segmentation techniques depend on the selection of initial regions, managing complex control structures, to obtain region boundaries which are often distorted, so that a merge step is needed to provide the final segmentation [22].

In our approach we identify an internal region by means of a seed, which in turn is used by an adapted method based on partial differential equations (PDE), specifically the Eikonal equation. With good image enhancements, solving the Eikonal equation to find the connected regions of interest, is simplified. In particular, this method allows us to naturally eliminate regions of the images that are not relevant and to extract connected regions which corresponds to vessels. 
The region grows by moving its boundary with unitary normal velocity as long as the region is inside the vessel to be identified. We have to solve the Eikonal equation:

$$
\begin{aligned}
& |\nabla T(x)|=\frac{1}{V(x)}, \quad x \in \Omega, \\
& T(x)=0, \quad x \in \Omega_{0} \subset \Omega,
\end{aligned}
$$

where $\Omega_{0}$ is the starting region included in a vessel and the variable $T$ corresponds to the arrival time of the interface of the growing region. The normal velocity $V$ ranges from 1 , where the image is white, to a value close to 0 , where the image is black. The identified connected region corresponds to points where the arrival-time is finite. The algorithm ends when $T$ reaches the size of the image. Furthermore, several connected regions can be identified by starting with an adapted initial region defined by the seed.

The Eikonal equation is solved by the fast marching method introduced by James A. Sethian [11]. The information propagates outward from the interface and allows a local solver of the Eikonal equation. With an adapted heapsort algorithm, the resolution is obtained in a sufficiently short time for an image of 1 megapixels, with a cost of $O(n \log n)$ where $n$ is the number of pixels. For a computation on the full domain, the Eikonal equation is solved sequentially in approximatively 10 seconds on a laptop. A classical flood fill method is slightly less costly, i.e. a linear cost, but the accurate resolution of the Eikonal equation allows us to compute the two-dimensional distance function $\psi$ to the boundary of each region. This technique is close to the one developed in [29] where the distance function computation in each point is increased with the boundary point it came from, as the front advances.

\subsection{Smoothing of the $2 D$ level set data}

Once every vessel is identified, the Eikonal equation is solved again to compute the signed distance function to the interface of vessels. The distance function to the vessels includes the information of the noisy image and pixelation effects induce high frequencies in the distance function. Image enhancement will be added by filtering high frequencies. An average value with five points (a pixel and its four neighbhours) fulfill this function and corresponds to an approximation of the heat equation up to the final time $\frac{\mathrm{h}^{2}}{5}$ where $h$ is the pixel size. With such a small final time, high frequencies are damped and low frequencies are slightly modified. If $\phi_{0}$ is the initial data, then:

$$
\phi_{0}^{\ell}(i, j)=\frac{1}{5}\left(\phi_{0}(i, j)+\phi_{0}(i-1, j)+\phi_{0}(i+1, j)+\phi_{0}(i, j-1)+\phi_{0}(i, j+1)\right),
$$

where $\phi_{0}^{\ell}$ is the $2 D$ level set function obtained after smoothing. The diffusion phenomenon induces a mass loss. For this reason, another algorithm will take place to provide the conservation of the mass. Let $S_{0}$ be the initial surface defined by $\left(\phi_{0} \leq 0\right)$ and $S_{0}^{\ell}$ defined by $\left(\phi_{0}^{\ell} \leq 0\right)$ :

$$
S_{0}=\int_{\Omega} H^{\epsilon}\left(-\phi_{0}\right) d x \quad \text { and } \quad S_{0}^{\ell}=\int_{\Omega} H^{\epsilon}\left(-\phi_{0}^{\ell}\right) d x
$$


where $H^{\epsilon}$ is the Heaviside regularized function. The algorithm for the mass correction modify $\phi^{\ell}$ until the desired mass $S_{0}$ is reached: let us iterate on $n$ the following assignment

$$
\left\{\begin{array}{l}
\phi_{n}^{\ell}(i, j)=\frac{1}{5}\left(\phi_{n}(i, j)+\phi_{n}(i-1, j)+\phi_{n}(i+1, j)+\phi_{n}(i, j-1)+\phi_{n}(i, j+1)\right), \\
S_{n}^{\ell}=\int_{\Omega} H^{\epsilon}\left(-\phi_{n}^{\ell}\right) d x \\
\phi_{n+1}=\phi_{n}^{\ell}+\frac{\varepsilon}{P_{0}}\left(S_{n}^{\ell}-S_{0}\right),
\end{array}\right.
$$

where $P_{0}$ is the perimeter of the level $\phi_{0}=0$ and $\varepsilon$ is chosen small enough $(\varepsilon=0.1)$ in order to define a convergent algorithm. Note that the perimeter is computed as:

$$
P_{0}=\int_{\Omega} \delta^{\epsilon}\left(-\phi_{0}^{\ell}\right) d x
$$

where $\delta^{\epsilon}$ is the Dirac regularized function, and $\epsilon$ is the smoothing parameter which defines the fictive thickness of the interface [32] which is fixed at $\frac{3}{2} h$, where $h$ is the pixel size.

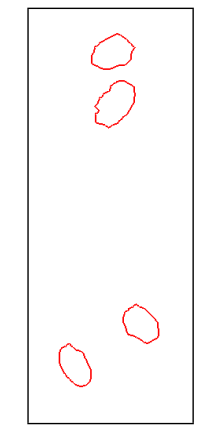

before smoothing

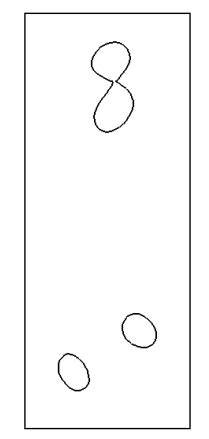

after smoothing

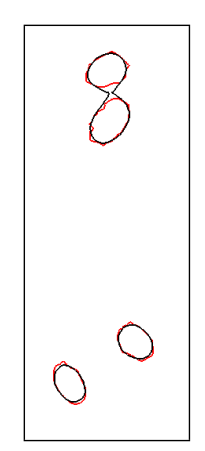

comparaison

Figure 2: Fusion with smoothing on global distance function.

If the smoothing algorithm proposed here is used with three to five iterations, high frequencies are damped and the interface is smooth. Nevertheless, if two connected components are close (with a distance less than five pixels), then the proposed algorithm will merge the two connected components as on Figure 2. This is due to the fact that the gradient of the distance function is singular between the two connected components. The successive values $\phi_{n}$ in (1.2) are then strongly modified in such a region.

For this reason, we have decided to apply the smoothing algorithm (1.2) on each connected component separately in order to smooth only the high frequencies due to noisy interfaces. We then compute the signed distance functions to the interface, called $\left(\phi_{0}^{C}\right)$. The obtained result after four iterations leads to the desired result shown on Figure 3, without fusion of the components. To reduce the computational time, the distance function is evaluated 30 pixels around the interface. On images of one megapixel, the vessels' diameter is a few tens of pixels which explains a drastic 
saving time between the full computation (13 seconds) and the computation reduced to 30 pixels $\left(6.10^{-2}\right.$ seconds).

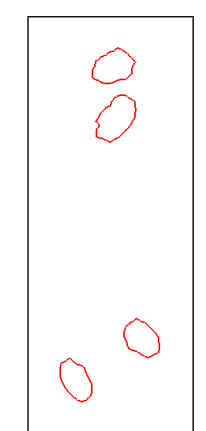

before smoothing

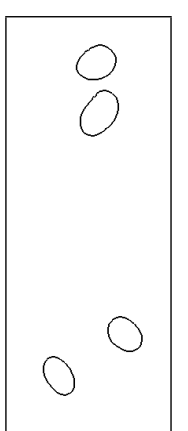

after smoothing

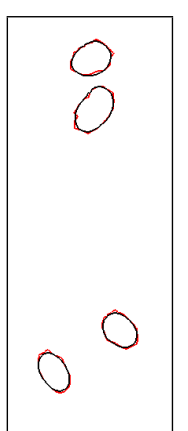

comparaison

Figure 3: No fusion smoothing with componentwise distance function.

After applying the algorithm of smoothing (1.2) on the level set functions $\left(\phi_{0}^{C}\right)$, we obtain the level set functions $\left(\phi_{\ell}^{C}\right)$ associated to the existing connected components on each cut. The relative mass error, after our algorithm, is small, about $0.1 \%$ on the example of Figure 3. Note that the mass (or surface) of a vessel, $S^{C}$, associated to the connected component $C$, is computed with the following formula:

$$
S^{C}=\int_{\Omega} H^{\epsilon}\left(-\phi_{0}^{C}\right) d x
$$

where $H^{\epsilon}$ is the Heaviside regularized function, and $\epsilon$ is the smoothing parameter defining the fictive thickness of the interface. It is chosen with a size of 1 to 2 pixels [32].

Centers of connected components on $2 D$ cuts From the level set functions $\phi_{\ell}^{C}$, we can determine the center of each connected component $C$ on each cut by the following formula:

$$
\overrightarrow{X^{C}}=\frac{\int_{\Omega} \overrightarrow{X^{C}} H^{\epsilon}\left(-\phi_{\ell}^{C}\right) d \vec{X}}{\int_{\Omega} H^{\epsilon}\left(-\phi_{\ell}^{C}\right) d \vec{X}}
$$

where $\overrightarrow{X^{C}}=\left(x^{C}, y^{C}\right)$.

\section{Construction of geometry graph $(G)$}

In this part, we consider that vessels are known on the transversal cuts. For two successive transversal cuts, the centers and the surfaces of the vessels are known (subsection 1.5). The difficulty is to connect centers of vessels between two successive transversal cuts in order to produce a graph corresponding to the network of vessels without additional information. The graph will be chosen as an optimal graph of an optimization problem to define. This formalism is known as the MongeKantorovich problem [10]. Let us give two measures $\mu$ and $\nu$ on two successive transversal cuts $X$ 
and $Y$ of $\mathbb{R}^{2}$, these measures are finite sums of atomic measures located on the centers of vessels:

$$
\mu=\sum_{i=1}^{k} m_{i} \delta_{x_{i}} \quad \nu=\sum_{j=1}^{\ell} n_{j} \delta_{y_{j}}
$$

The mass of each atomic measure is related to the surface of the vessels. The Monge-Kantorovich problem impose that

$$
\mu(X)=\nu(Y)
$$

Following [34] we define a transport path that carries $\mu$ to $\nu$ as a weighted directed graph $(G)$ which vertices contains the points $\left(x_{i}\right)$ and $\left(y_{j}\right)$ and a mass function

$$
w: E(G) \longrightarrow(0,+\infty),
$$

where $E(G)$ is the set of the directed edges of $(G)$. Moreover, for all vertex $v$ of $(G)$, the mass $w$ satisfies the Kirchhoff's law:

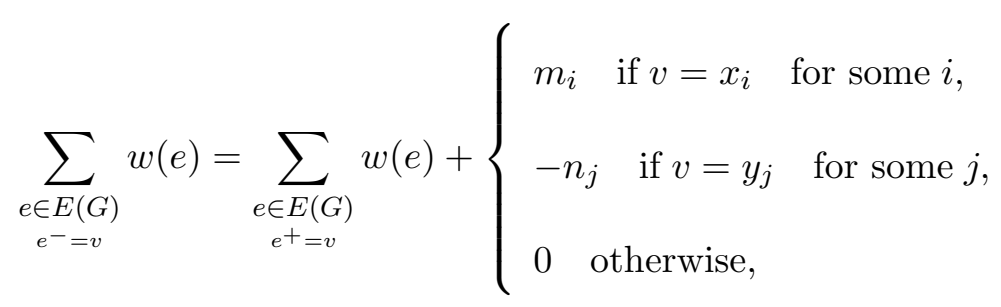

where $e^{-}$and $e^{+}$denote the starting and the ending points of each directed edges of $e \in E(G)$.

Denote

$$
\operatorname{path}(\mu, \nu)=\{\text { all transport paths from } \mu \text { to } \nu\}
$$

and

$$
\mathcal{G}=\bigcup_{X=\operatorname{supp}(\mu), Y=\operatorname{supp}(\nu)} \operatorname{path}(\mu, \nu),
$$

the union of all transport paths between the two measures $\mu$ and $\nu$ of the two successive cuts $X$ and $Y$ verifying the mass constraint (2.1). Among all paths in path $(\mu, \nu)$, we want to find an optimal path defining the graph $(G)$, by minimizing the following cost function referred as the Gilbert-Steiner problem [9]:

$$
\min _{G \in \mathcal{G}} M(G) \quad \text { such that } \quad M(G)=\sum_{e \in E(G)} \omega(e)^{\alpha} l(e)
$$

where $\alpha<1, l(e)$ is the length of the edge $e$ of the graph $(G)$ and $w(e)$ is the mass transported along the edge $e$. The choice of a cost function with $\alpha<1$ is able to enforce the reconnection of branches, which is known as the "branched transport" problem [9], [34], [21] and [2].

In [21] the authors propose an approximation based on a regularization of the non-convex functional with an additional quadratic term [18]. When the quadratic term is sufficiently large, the functional is convex. Then, a gradient method can be applied and converge to the minimum. By decreasing incrementally the value of the parameter $\epsilon$ (the coefficient of the quadratic term), a new optimal solution is solved. The minimum point found in the previous step becomes the 
initialization for the new fonctional. This method obliges to implement a global gradient method whereas an other approach [34] permits to use a simpler algorithm based on a sequence of local optimization problems. The local problem is the Gilbert-Steiner problem with three points, which admits an analytic solution.

So, to minimize the non-convex functional (2.3), we are interested in the work of Xia [34] (developped in 2.2). As in the approach of Oudet [21], Xia does not give any warranty for obtaining the global minimum, but a low functional cost is identified. Because the choice of the cost function is arbitrary and because the reconstruction depends on the choice of the position of the transversal cuts, an exact identification of the minimum is not required. The optimization only serves to compare the different possible reconnections. A pertinent graph $(G)$ is then selected.

In the following subsections, we construct the transport measures on two successive cuts and then we study Xia's work [34] for obtaining the graph connecting the vessels on these two cuts.

\subsection{Construction of the transport measures}

In the first section, we are able to extract the vessel contours on transversal cuts. By a human intervention, the vessels are identified on the image with their centers and surfaces which are related to the transported masses. The raw data on the surfaces, used as mass to transport, do not verify the constraint (2.1). The aim of this subsection is to construct the two measures $\mu$ and $\nu$ on two successive cuts. Firstly, we associate to the measure $\mu$ (respectively to the measure $\nu$ ), atomic measures located at the centers of the vessels for the cut $X$ (respectively for the cut $Y$ ). The mass of each atomic measure has to be defined and is chosen homogeneous to a flow. From the Murray law [20], the velocity of the blood flow is homogeneous to the radius of the vessel so that the theoretical flow $d$ is defined as

$$
d=S^{\frac{3}{2}}
$$

where $S$ is the surface to the cut of the vessel. We denote by $\tilde{m}_{i}$ (resp. $\tilde{n}_{i}$ ) the theoretical flow associated to the $i$-th atomic measure of $\tilde{\mu}$ (resp. $\tilde{\nu}$ ):

$$
\tilde{\mu}=\sum_{i=1}^{k} \tilde{m}_{i} \delta_{x_{i}} \quad \tilde{\nu}=\sum_{j=1}^{\ell} \tilde{n_{j}} \delta_{y_{j}} .
$$

The measures $\tilde{\mu}$ and $\tilde{\nu}$ do not verify (2.1) and are then modified as follows. Let us minimize the following functional defined by:

$$
F(d)=\sum_{i=1}^{k}\left(m_{i}-\tilde{m}_{i}\right)^{2}+\sum_{j=1}^{\ell}\left(n_{j}-\tilde{n}_{j}\right)^{2}
$$

for all $m=\left(m_{1}, \cdots, m_{k}\right)$ and $n=\left(n_{1}, \cdots, n_{\ell}\right)$ such that

$$
\sum_{i=1}^{k} m_{i}=\sum_{j=1}^{\ell} n_{j}
$$

We then obtain the corrected measures $\mu$ and $\nu$

$$
\mu=\sum_{i=1}^{k} m_{i} \delta_{x_{i}} \quad \nu=\sum_{j=1}^{\ell} n_{j} \delta_{y_{j}},
$$


verifying (2.1) thanks to (2.5). In the next subsections, we can minimize the cost function (2.3) in order to obtain the optimal graph that moves $\mu$ to $\nu$.

\subsection{Minimization for mass transfer problem}

In the previous subsection, we have constructed the two measures $\mu$ and $\nu$ on the two successive cuts $X$ and $Y$. The aim of this subsection is to connect the centers of vessels between the cuts by an optimal path which defines the graph. For this reason, we are interested in the work of Xia [34] who proposed a succession of local optimization of connections to three points studied by Gilbert and Steiner in [9]. The problem consists to minimize the cost of networks supporting a given set of masses between cuts, defined in (2.3).

\subsubsection{Case of the graph of two sources to one target}

Let $A_{1}, A_{2}$ be two points of the cut $X$ and let $A_{3}$ be a point of the cut $Y$. We set: $\mu=m_{A_{1}} \delta_{A_{1}}+$ $m_{A_{2}} \delta_{A_{2}}$ and $\nu=m_{A_{3}} \delta_{A_{3}}$, with $m_{A_{1}}+m_{A_{2}}=m_{A_{3}}$. The aim is to find the optimal path from $\mu$ to $\nu$ under the cost function (2.3). We give:

$l(e)=\left\|A_{i}-B\right\| \quad$ and $\quad \omega(e)=m_{A_{i}} \quad \forall i=\{1,2,3\}$, for $e=\left(A_{1}, B\right)$ or $e=\left(A_{2}, B\right)$ or $e=\left(B, A_{3}\right)$.

Then, the cost function used in this case is:

$$
F(B)=m_{A_{1}}^{\alpha}\left\|A_{1}-B\right\|+m_{A_{2}}^{\alpha}\left\|A_{2}-B\right\|+m_{A_{3}}^{\alpha}\left\|A_{3}-B\right\|
$$

$F$ must achieve its minimum at point $B^{\star}$. The bifurcation point $B^{\star}$ has to satisfy the optimal angles constraints:

$$
\theta_{1}=\widehat{A_{3} B^{\star} A_{1}}, \quad \theta_{2}=\widehat{A_{3} B^{\star} A_{2}} \quad \text { and } \quad \theta_{3}=\widehat{A_{1} B^{\star} A_{2}}
$$

for more details on obtaining the optimal angles see [2],[9],[34]. The point $B^{\star}$ is necessarily located in the interior of the triangle $\left(A_{1} A_{2} A_{3}\right)$, this condition is expressed as:

$$
\theta_{1}>\widehat{A_{1} A_{2} A_{3}}, \quad \theta_{2}>\widehat{A_{2} A_{1} A_{3}} \text { and } \theta_{3}=\theta_{1}+\theta_{2}>\widehat{A_{1} A_{3} A_{2}},
$$

and can be verified on the Figure 4. The path is then referred as a "Y-shaped" path.

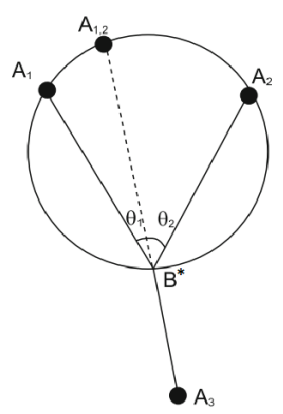

Figure 4: Optimal point $B^{\star}$ located inside the triangle $\left(A_{1} A_{2} A_{3}\right)$. 
If the conditions on the localization of the point $B^{\star}$ inside the triangle $\left(A_{1} A_{2} A_{3}\right)$ are not fulfilled, three degenerate cases are introduced in [9]:

- If $\widehat{A_{1} A_{3} A_{2}} \geq \theta_{3}$ then $B^{\star}=A_{3}$, the path is referred as a "V-shaped" path (see the Figure 5 (right)).

- If $\widehat{A_{1} A_{2} A_{3}} \geq \theta_{1}$ and $\widehat{A_{1} A_{3} A_{2}}<\theta_{3}$ then $B^{\star}=A_{2}$, the path is referred as a "L-shaped" path (see the Figure 6 (left)).

- If $\widehat{A_{2} A_{1} A_{3}} \geq \theta_{2}$ and $\widehat{A_{1} A_{3} A_{2}}<\theta_{3}$ then $B^{\star}=A_{1}$, the path is referred as a "L-shaped" path (see the Figure 6 (right)).

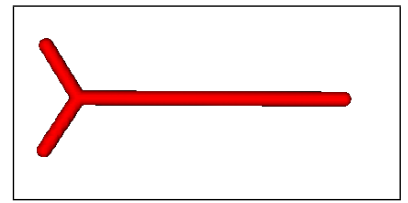

$\alpha \approx 0$

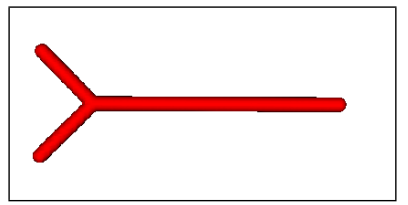

$\alpha=0.5$

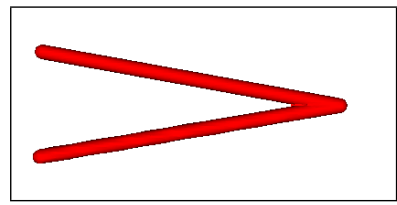

$\alpha \approx 1$

Figure 5: Optimal irrigations leading to "V-shaped" and "Y-shaped" paths.
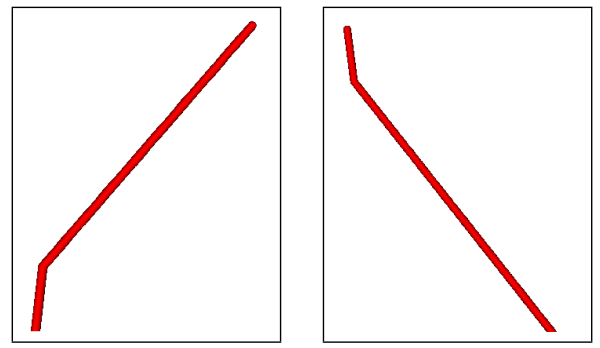

Figure 6: Two constructions of L-shaped paths.

\subsubsection{Optimization over three nodes: sources or targets}

In this part, we treat the case where $n=k+\ell>3$ to minimize the non-convex cost function (2.3). To solve this minimization problem, Xia proposed in [34] a method based on successive optimization with three nodes of the graph as in subsection 2.2.1. The graph is first initialized with a maximal ramification: it is constituted with two binary trees $T_{\mu}, T_{\nu}$ connected by their root. A binary tree is constructed from leaves $x_{i}$ (resp. $y_{j}$ ), associated to measure $\mu$ (resp. $\nu$ ) as drawn on the Figure 7. The pairs of leaves are associated by a closeness criteria. 

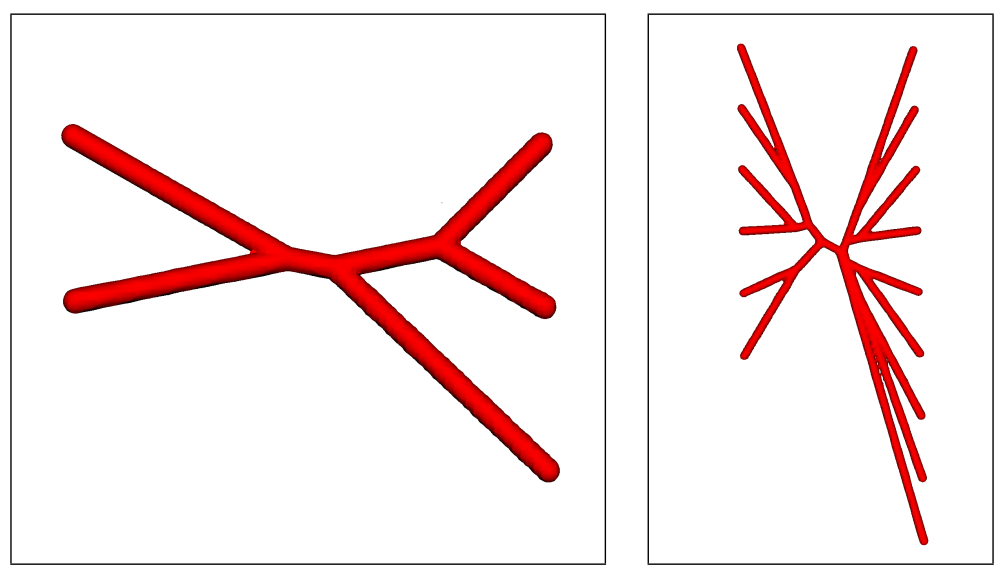

Figure 7: Construction of the initial graphs.

Visiting the nodes of the initial graph, we get rid of unnecessary nodes (e.g. some node may have only one child and one parent). Then, the position of the interior nodes is locally optimized as above in section 2.2, where we study the local structure with two sources and one target. However, the optimization does not stop here because Xia has proposed to change the structure of the graph if a node $v$ has two children $v_{c h_{1}}$ and $v_{c h_{2}}$, one parent $v_{p}$ who has two parents $v_{p p_{1}}$ and $v_{p p_{2}}$ (see the Figure 8).
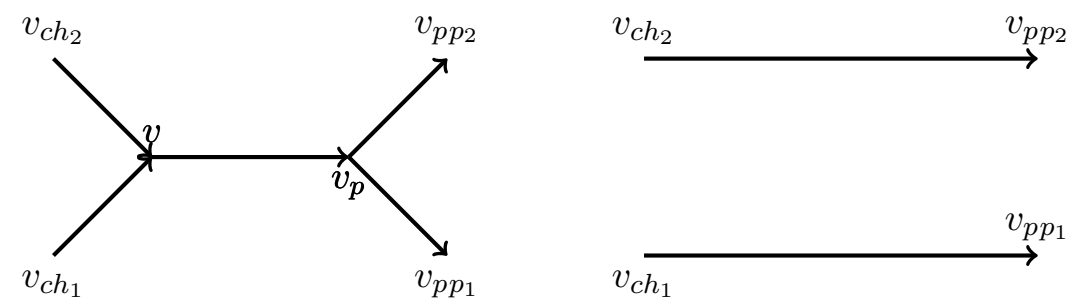

Figure 8: Representation of a H-shaped path (left) and its modified path (right).

This last step, which modifies the topology of the graph, is applied if it reduces the transportation cost. Applying the topology change, the graph always keeps the local structure of two sources and one target or one source and one target (see the Figures 9,10). The optimization process proposed is repeated until it converges to an optimal graph. 

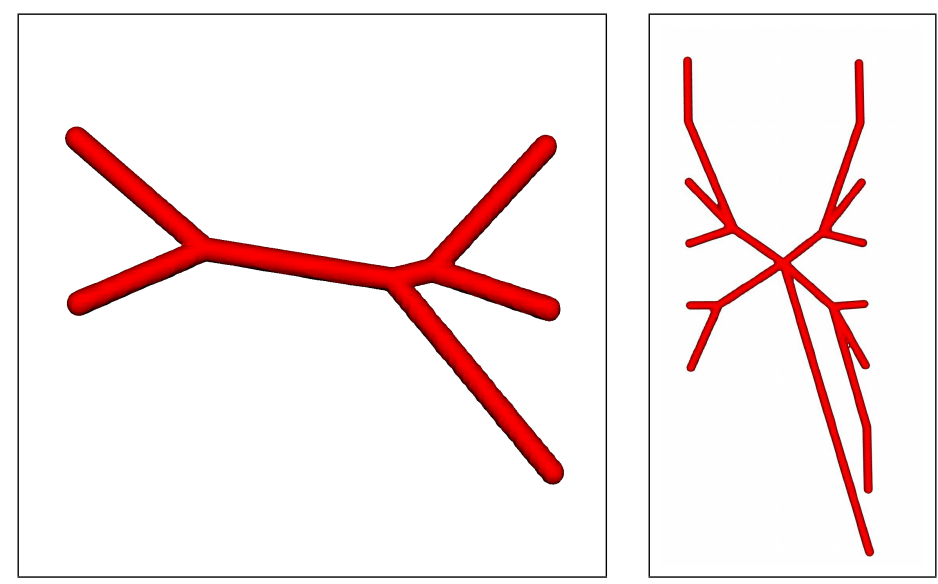

Figure 9: Optimization of graphs without the topology change for $\alpha=0.75$.
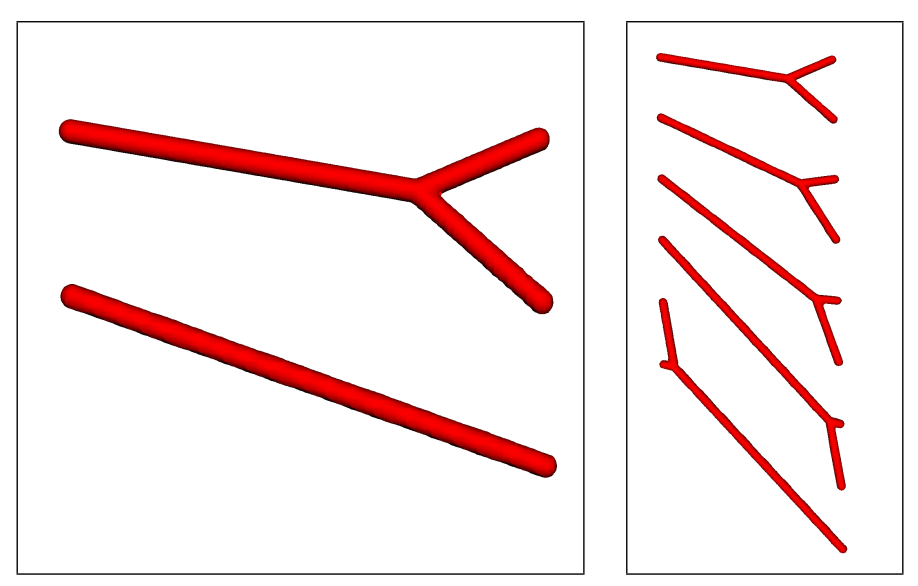

Figure 10: Optimization of graphs with the topology change for $\alpha=0.75$.

We observe that the graph is nearly converged in few iterations (see the Figures 11, 12 (right)). The topology change completely modifies the structure of the graph (see the Figures 9,10), and reduces the cost to transport $n=k+\ell>3$ atomic measures between two successive transversal cuts $X$ and $Y$ (see the Table 1) and (see the Figures 11, 12 (left)). 


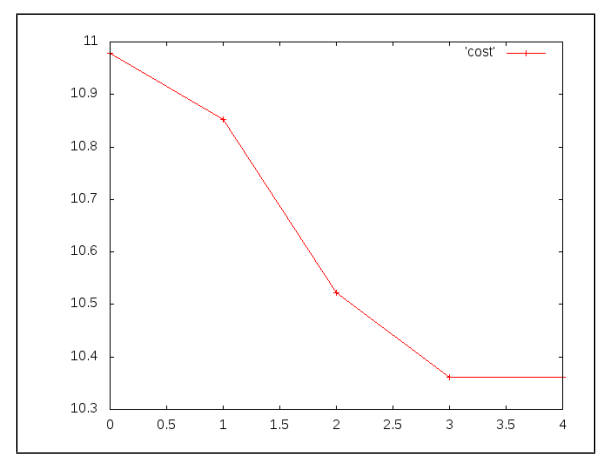

with the topology change

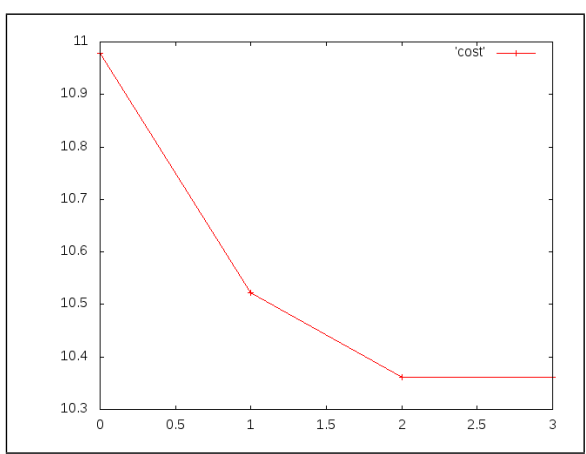

without the topology change

Figure 11: Variation of the cost function to irrigate three targets with two sources for $\alpha=0.75$.

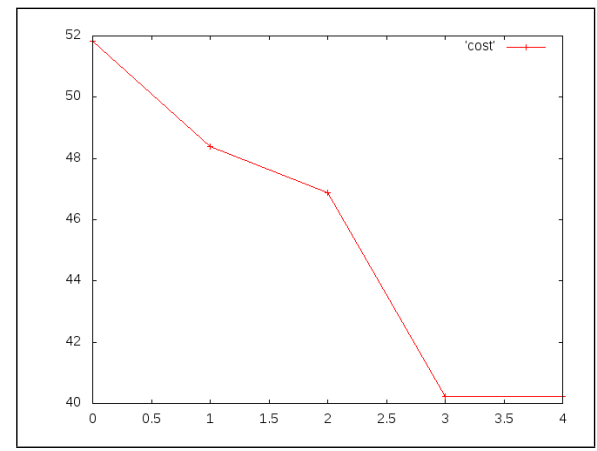

with the topology change

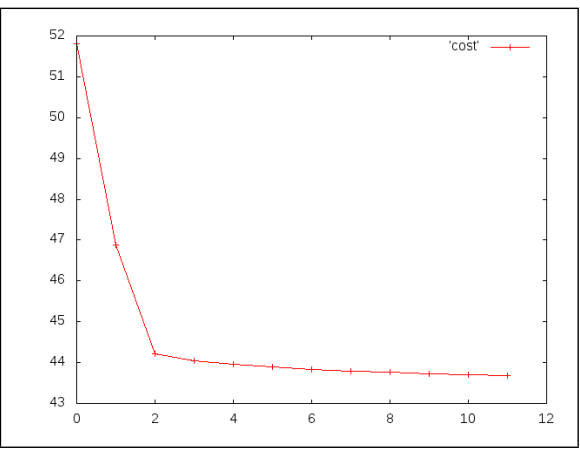

without the topology change

Figure 12: Variation of the cost function to irrigate nine targets with six sources for $\alpha=0.75$.

\begin{tabular}{|c|c|c|c|c|c|}
\hline$n$ & $m$ & $\alpha$ & the cost before the optimization & \multicolumn{2}{|c|}{ the cost after the optimization } \\
\hline & & & & without the topology change & with the topology change \\
\hline 2 & 3 & 0.75 & 10.977 & 10.840 & 10.360 \\
\hline 6 & 9 & 0.75 & 51.815 & 46.081 & 40.982 \\
\hline
\end{tabular}

Table 1: The cost of transport before and after optimization.

Xia's tool is inexpensive in computation time and is relatively simple to implement. Furthermore, it selects a graphical configuration with an important reduction of the cost between the initial graph which is strongly ramified and the optimal graph obtained after the optimization process. 
The next subsection is devoted to the conservation of the masses into each bifurcation points which allows a reconstruction of the vessels on these points.

\subsection{Standardized surface variations on the graph $(G)$}

In the previous subsection, we have optimized the graph $(G)$ of the vessels in order to connect them. To design the shape of the vessels, the vessel size must vary slowly along the graph $(G)$ and verify some properties at bifurcation point. For this reason, we tend to minimize variation of a fictive flow along the graph. Let $\theta(x)$ be the fictive flow associated to a section of the vessel at any point $x$ of the graph $(G)$. The goal is to minimize:

$$
\int_{\mathrm{G}}|\nabla \theta(\mathrm{x})|^{2} \mathrm{dx}
$$

The minimization of the equation (2.7) is made under the following constraints:

- The Murray law [20] is imposed on each bifurcation point. In Murray's optimum system, the flow and the vessel radius are functionally related: an optimum radius is found for a given flow. The volume of a vascular system will depend upon the flow required: an optimum vasculature for high flows will have larger vessels than one for low flows, the cubes of the vessel radius is proportional to the flows required $\left(\theta(x)=r(x)^{3}\right)$.

- The flow $\theta$ is imposed on the transversal cuts from the transversal surface of vessels, projected on normal section of such vessels, denoted by $S_{p}$.

After the minimization of (2.7), the flow varies linearly on each branch and is then defined by its values on extremities of each branch $e=\left(e^{-}, e^{+}\right)$of the graph $(G)$ denoted by

$$
\theta\left(e^{-}\right)=S_{p}^{\frac{3}{2}}\left(e^{-}\right) \text {and } \theta\left(e^{+}\right)=S_{p}^{\frac{3}{2}}\left(e^{+}\right) .
$$

One can see, from the obtained result in the Figure 13, that the variation of the surfaces is achieved along the branches in a gentle manner for three examples (two sources to three targets, two sources to four targets and one source to four targets).
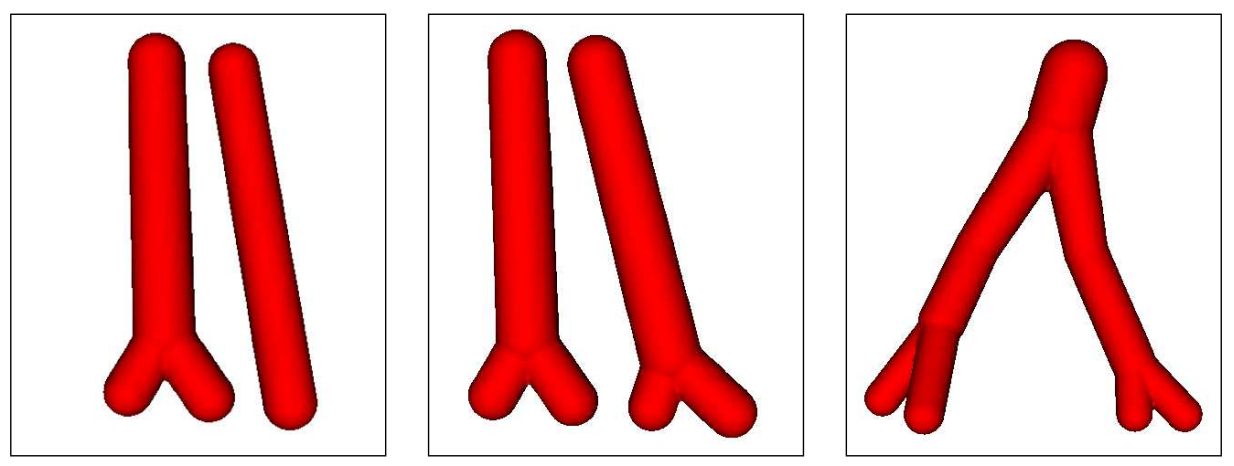

Figure 13: The reconstructed vessels after radius optimization.

The next section is devoted to the construction of the global geometry by using information of the constructed graph $(G)$ and data on $2 D$ transversal cuts. 


\section{Complete reconstruction of the $3 D$ geometry}

To achieve the complete reconstruction of blood vessels for the abdominal aorta and for the femoral artery, we first construct the graphes associated to images of scanners. The reconstruction of the $3 D$ level set function, which is the $3 D$ geometry of the abdominal aorta (Figure 15) or the femoral artery (Figure 16), is made by using the $2 D$ level sets functions given by the images and the graphs information (Figure 14) as follows:

Let $\left(e=\left[e^{-}, e^{+}\right]\right)$be a branch of the graph $(G)$, with $e^{+/-}=\left(e_{1}^{+/-}, e_{2}^{+/-}, e_{3}^{+/-}\right)$in the coordinate system whose third direction is normal to transversal cut. Let $\phi_{e}(x)$ be the level set function attached to the branch $e$ at the point $x \in \mathbb{R}^{3}$ for every $e_{3}^{-} \leq x_{3} \leq e_{3}^{+}$, defined by the directional interpolation

$$
\phi_{e}\left(\lambda e^{-}+(1-\lambda) e^{+}\right)=\lambda \phi\left(e^{-}\right)+(1-\lambda) \phi\left(e^{+}\right),
$$

where $\lambda=\frac{x_{3}-e_{3}^{-}}{e_{3}^{+}-e_{3}^{-}}$and where $\phi\left(e^{+/-}\right)$is known from data imagery or from an analytic computation

$$
\phi\left(e^{+/-}\right)=d\left(e^{+/-}, x^{+/-}\right)-R_{e^{+/-}},
$$

where $R_{e^{+/-}}$is the radius associated to the surface $S_{p}\left(e^{+/-}\right)$defined in $(2.8)$ and the points $x^{+/-}$ belongs to the same plan, normal to the third direction, than $e^{+/-}$. Furthermore, the point $x$ belongs to the line $\left(x^{-}, x^{+}\right)$parallel to $\left(e^{-}, e^{+}\right)$. Then, the $3 D$ level set function is obtained as follows :

$$
\varphi(x)=\min _{e \in E(G)} \phi_{e}(x)
$$

To correct the singularities of the level set function $\varphi$ near a bifurcation point $v$ of the graph $(G)$, we introduce the spherical $3 D$ level set function $\varphi_{G}$ :

$$
\varphi_{G}(x)=d(x, v)-\max _{e \in\left\{E(G) / v=e^{+/-}\right\}}\left(R_{e}\left(e^{+/-}\right)\right),
$$

where $R_{e}\left(e^{+/-}\right)$is the radius associated to the surface $S_{p}\left(e^{+/-}\right)$defined in $(2.8)$. Finally, the $3 D$ level set functions between two successive transversal cuts for all points $x \in \mathbb{R}^{3}$ is given by:

$$
\psi(x)=\min \left(\varphi(x), \varphi_{G}(x)\right)
$$

In order to reconstruct the level set function $\psi$ on a mesh of our choice with a reduced computational cost, an iterative process is employed:

- Computation of the level set function $\psi$ on a coarsed cartesian grid limited by the first and last cut.

- Computation of the level set function $\psi$ on a cartesian subgrid (three times thiner) of the previous mesh restricted to points where $|\psi| \leq \epsilon$. In order to add the mesh of the interior of the geometry, we replace $|\psi| \leq \epsilon$ with $\psi \leq \epsilon$.

- Iterate while the desired fine grid is not reached.

Remark 3.1 Note that for branches with very low flow, which appear only to equilibrate flow but do not correspond to vessels, the construction of the level set function passes over such branches. 


\section{Numerical validation}

The aim of this section is to validate the numerical codes that achieve the $3 D$ reconstruction of blood vessels from a limited number of transversal cuts. This validation is performed by the transversal cuts of the abdominal aorta and the femoral artery. For this reason, we construct the global graph which connects the centers of the blood vessels between the successive cuts by using the approach described in 2 in order to produce the graphs of the abdominal aorta and the femoral artery (Figure 14). Finally, we reconstruct the $3 D$ level set of the abdominal aorta (respectively of the femoral artery) as in section 3 (see Figures 15, 16).

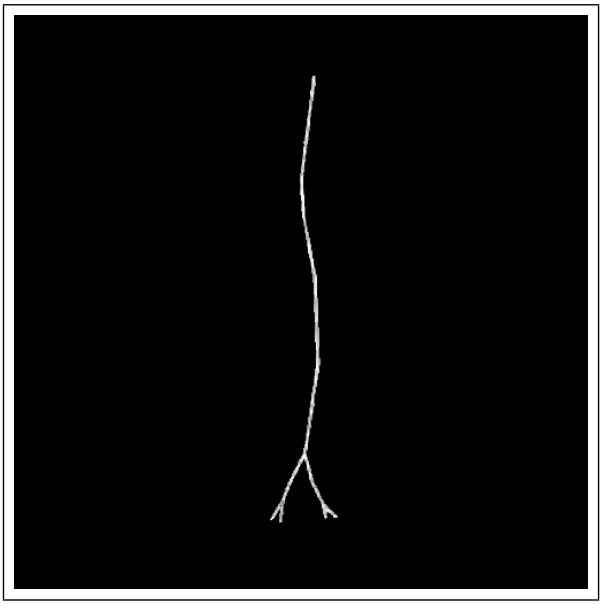

abdominal aorta

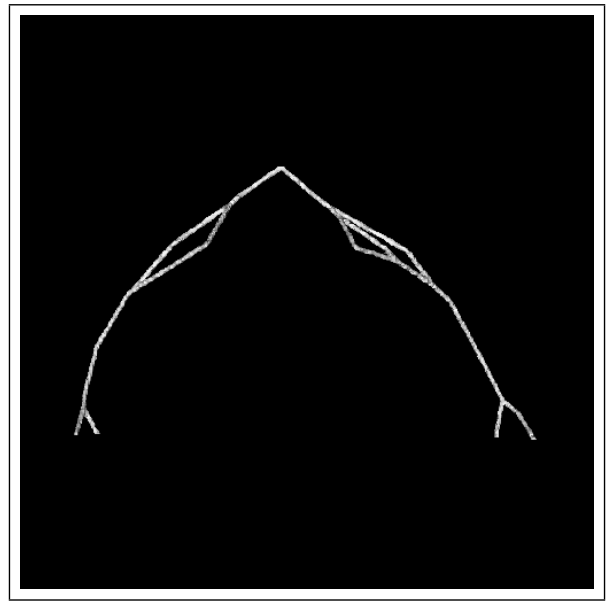

femoral artery

Figure 14: The $3 D$ graphs of the geometries.
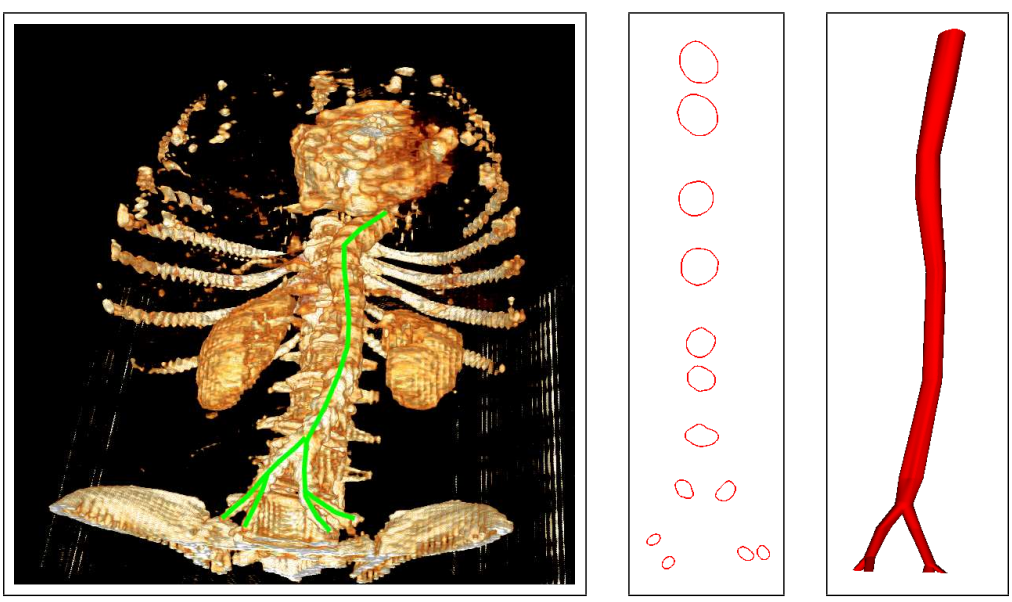

Figure 15: The $3 D$ image of abdominal aorta on the left. The $2 D$ superposition of vessel contours related to abdominal scanners on the center. The $3 D$ reconstruction from vessel contours of the abdominal aorta on the right. 

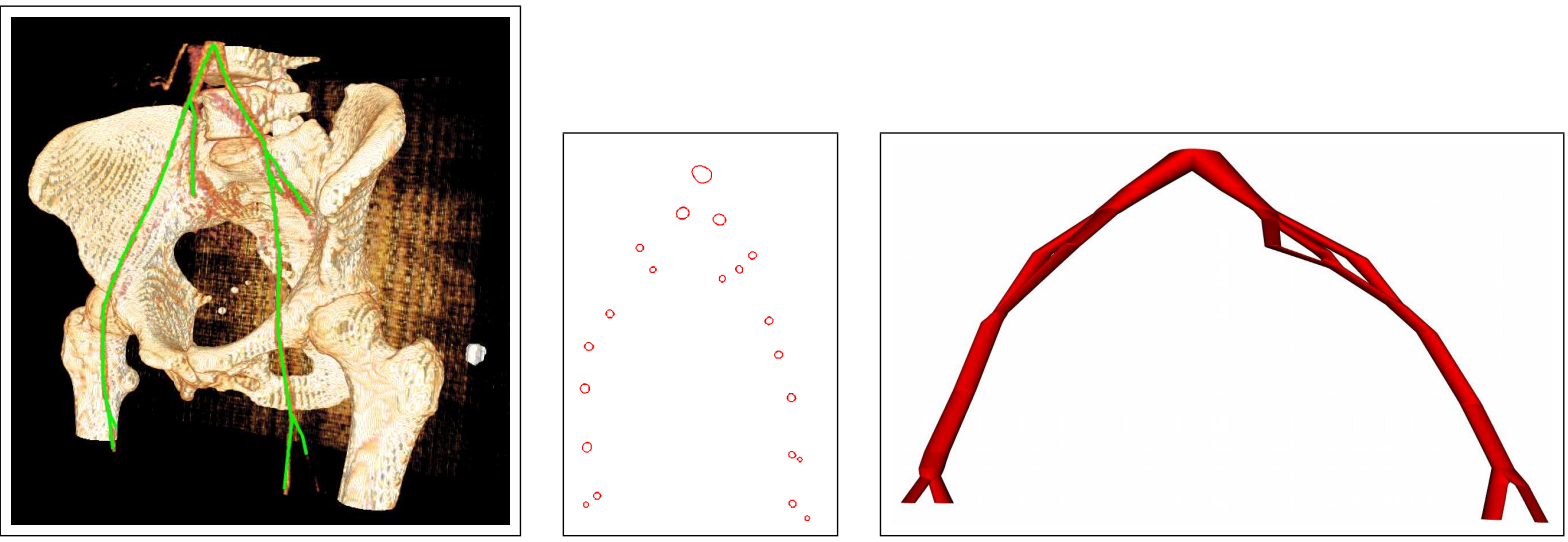

Figure 16: The $3 D$ image of femoral artery on the left. The $2 D$ superposition of vessel contours related to femoral scanners on the center. The $3 D$ reconstruction from vessel contours of the femoral artery on the right.

The reconstruction of the femoral artery connects the whole cuts. The artery that finishes its race is therefore artificially reconnected to other arteries (Figure 16). A specific treatment is needed to handle this case.

\section{$5 \quad$ Enrichment with new $2 D$ cuts}

In the previous sections, we succeeded in achieving $3 D$ reconstruction of blood vessels from a limited number of organ cuts. A human intervention is necessary to identify regions corresponding to vessels. The $3 D$ reconstruction has produced the first approximation of vessels but, without human intervention, it is necessary to include new $2 D$ cuts on the $3 D$ reconstruction. The difficulty is to identify and extract the vessels in the new cuts. Then we search for interfaces on the $2 D$ images close to the predicted vessel initially reconstructed.

In [13] it is introduced the concept of active contour model, also called snakes, which is a dynamic structure used in image processing and computer vision (it is introduced formally by Kass and Witkin in 1987). Active contour model is described by a curve which minimizes the energy outcome of external and internal forces. The external energy is supposed to be minimal when the snake is at the object boundary position. The internal energy is supposed to be minimal when the snake has a shape which is supposed to be relevant considering the shape of the final object. The snakes model is popular in computer vision, and led to several developments in $2 D$ and $3 D$.

Another technique image segmentation model was proposed by Mumford and Shah [19]. The Mumford Shah technique is the source of the region-based active contours, and it follows:

$$
F(u, \Gamma)=\mu^{2} \iint_{\Omega}(u-I)^{2} d x d y+\iint_{\Omega / \Gamma}\|\nabla u\|^{2} d x d y+\nu L(\Gamma)
$$

Like before, $I$ is our image function. We have $\Omega=\Omega_{1} \cup \Omega_{2} \cup \ldots \cup \Omega_{k} \cup \Gamma$ in which $\Omega$ is the domain of our image, $\Omega_{i}$ is the region in our image that represents object $O_{i}$ which does not include the boundaries, and $\Gamma$ is the set of smooth arcs that makes up boundaries for the $\Omega_{i} . L(\Gamma)$ is the total 
length of all the smooth arcs in the set $\Gamma$. The function $u$ is a function that is differentiable on $\Omega_{i}$ such that $1 \leq i \leq k$, but can be discontinuous across $\Gamma$. The values of $\mu$ and $\nu$ are weighting factors that control the quality of approximation and coarseness of the segmentation. A large $\nu$ will result in fewer boundaries.

The goal is to find $u$ and $\Gamma$ so that $F$ is minimized. The first term makes $u$ close to $I$, the second term ensures that the regions $\Omega_{i}$ does not change drastically, and the third term makes the boundaries $\Gamma$ as short as possible. In this approach, the evolution of the curve $\Gamma$ remaining from the gradient of the image $I$. The objects not defined by the gradient are not detected. For this reason, Chan and Vese [4] proposed a new model based on the Mumford-Shah functional (5.1) and depending on the level set functions.

In our case, snakes techniques [13] are not well adapted without human intervention, because they are methods that require an initialization by a contour placed inside or outside the region corresponding to a vessel by example.

The Mumford-Shah methods [19] or the Chan-Vese methods [4] are not well adapted because we want to eliminate a large portion of the image which doesn't contain the vessels. A way to eliminate the spaced regions is to exploit the information of the interface previously predicted. In the Mumford-Shah cost function, the data $I$ can be modified according to the distance of the interface previously predicted.

The need to compute a distance function leads us to adopt the following approach, based only on the evaluation of distances. First, we start from the $2 D$ image of a new transversal cut and segment the image as detailed in section (1). Then, the signed distance function to discontinuities of segmented image is produced by solving the Eikonal equation [11].

We denote by $\phi_{\text {org }}$ the $2 D$ level set functions which are described by the organs (the red color in the Figure 17) and by the $2 D$ level set functions $\phi_{0}$ which are the first approach of the vessels reconstructed in the previous sections (the black color in the Figure 17).

The goal is to move interface $\left(\phi_{0}=0\right)$ on a subset of $\left(\phi_{\text {org }}=0\right)$ with minimal displacement. The following evolution equation moves the values of $\phi$ with velocity 1 or 0 :

$$
\left\{\begin{array}{l}
\partial_{t} \phi(x, t)-\left(H(\phi)-H\left(\phi_{\text {org }}\right)\right)=0 \\
\phi(0)=\phi_{0}
\end{array}\right.
$$

where $H$ is the heaviside function : $H(\phi)=\frac{\operatorname{sgn}(\phi)+1}{2}$.

The choice of the model is justified by the fact that the solution of (5.2) is stationary in regions where the two data $\phi$ and $\phi_{\text {org }}$ present a coherence of sign and makes evolve $\phi$ in the direction of $\phi_{\text {org }}$ otherwise. When a related region of $\left(\phi_{\text {org }} \leq 0\right)$ is reached by $(\phi \leq 0), \phi$ does not evolve in this region. Then, $H(\phi)$ is temporary stationary in the full domain as soon as the sign of $\phi$ is constant on each connected component. This constitutes the stopping criterion of the algorithm (Figure 17). 
The obtained function $\phi$ after the resolution of (5.2) (the blue color in the Figure 17) loses its property of signed distance function. So, we reinitialize the $2 D$ signed distance function $\phi$ by solving the Eikonal equation (with velocity 1 ) with the fast marching method introduced by James A. Sethian [11], on a neighborhood of 30 pixels.

After the detection of the vessels on the new cuts, we extend the graph $(G)$ with the new information obtained from the new cuts. The $3 D$ reconstruction of the vessels takes into account the enriched graph and the new imagery cuts.
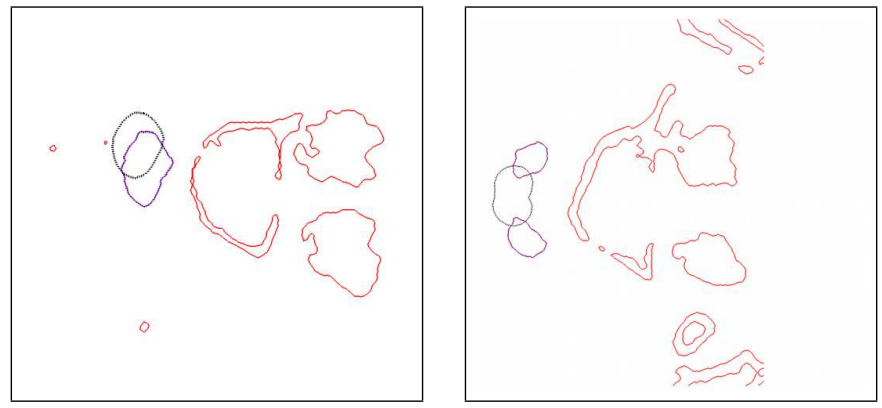

Figure 17: Two examples of the detection of vessel contours on the additional cuts.
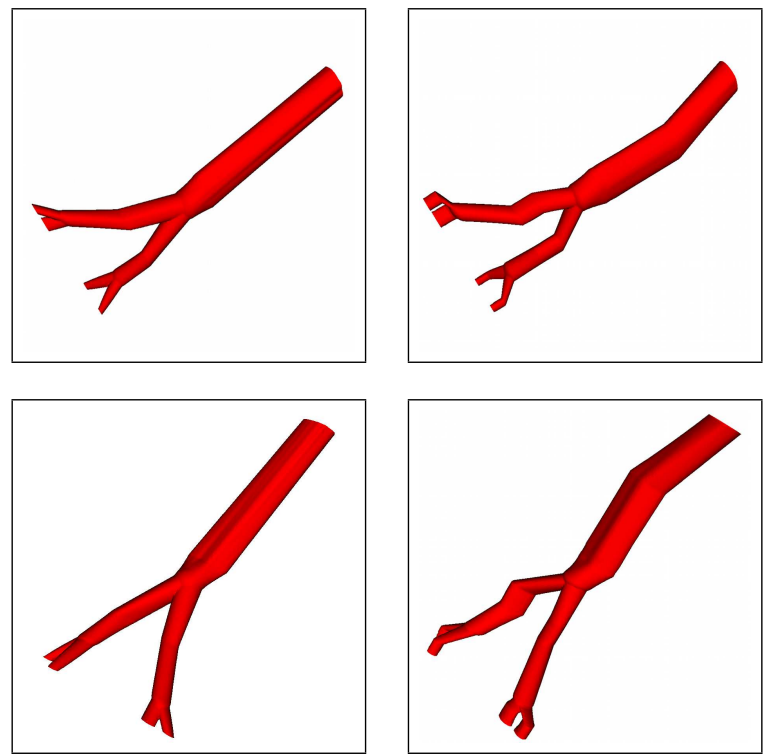

Figure 18: Two views of reconstruction with four cuts on the left, same views for the reconstruction enriched with nine additional cuts on the right. 

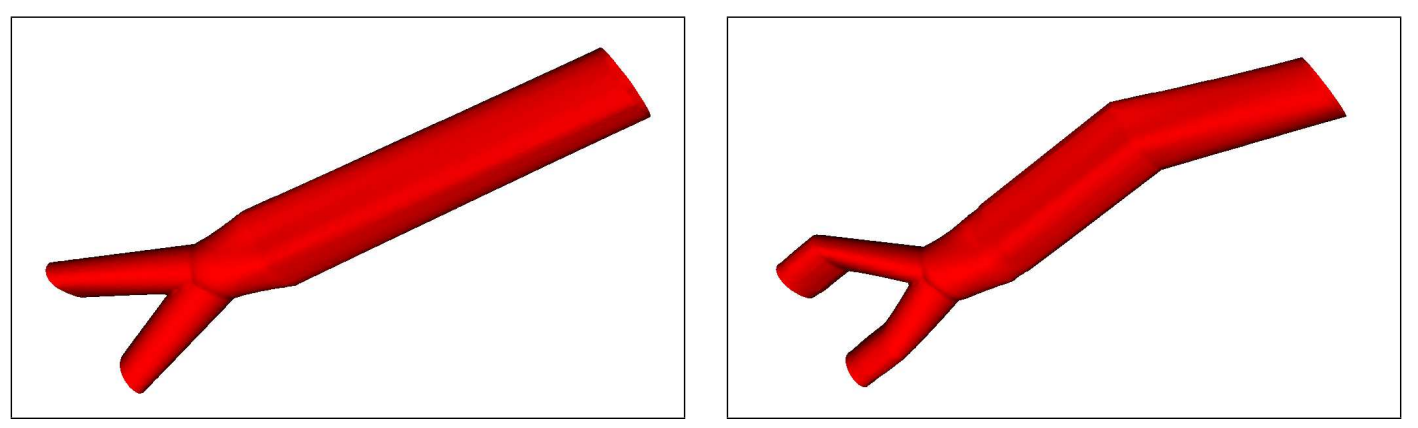

Figure 19: initial reconstruction with three cuts on the left, enrichment with six additional cuts on the right.

The enrichment corrects the positions of the arteries, but a limited enrichment leads to erroneous computations of transversal surface to the graph. The size of certain portions of arteries is flawed on the Figures 18 and 19, and less pertinent than the first reconstruction. If we have more data, it pays to use them. If the full data are available, another approach will be proposed in the following section.

\section{$63 D$ reconstruction from full data}

In the previous section, we succeeded in extending the geometry with new $2 D$ cuts using the information of the first reconstruction. In this section, the whole $2 D$ cuts are known, the $3 D$ reconstruction of vessels consists in extracting the relevant information. We use the previous tool developed in 5 to detect vessels close to the vessels of the previous cut.

For this, let us solve the equation (5.2), where $\phi_{0}$ is the level set function defining the vessels on the previous cut (the black color in the Figure 21) and $\phi_{\text {org }}$ is the level set function associated to the raw image. Then we obtain the function $\phi$ whose zero level, drawn with blue color, represents the interface of the vessel.

Afterwards we can apply, on each cut, the tools developed in subsection 1.2 and connect the centers of vessels, with a closeness criteria, between the successive cuts in order to produce the graph $\left(G^{\prime}\right)$ corresponding to the network of vessels. The $3 D$ reconstruction can then be applied as in section 3 by using the level set information on $2 D$ cuts and the precise graph $\left(G^{\prime}\right)$ (Figure 22).

The drawback of this method is that the whole data are necessary. Furthermore, the computational cost is important compare to the previous work, because it assumes a processing step on each cut. But it allows a fine reconstruction with a minimal human intervention on the first cut. 


\section{: \\ $0^{0}$}

Figure 20: The first cut acts as an information.

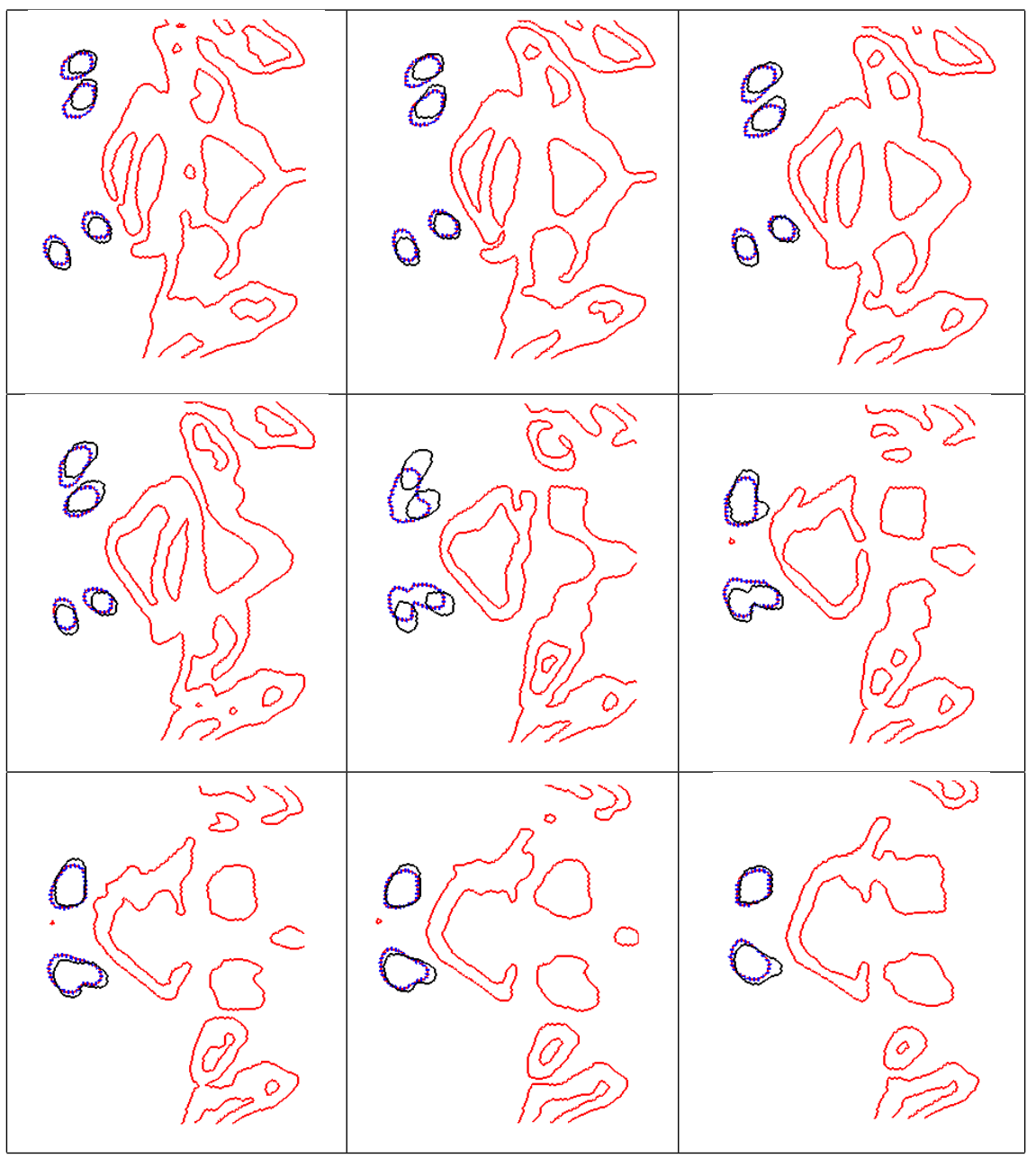

Figure 21: Detection of the blood vessel contours on all successive cuts. 

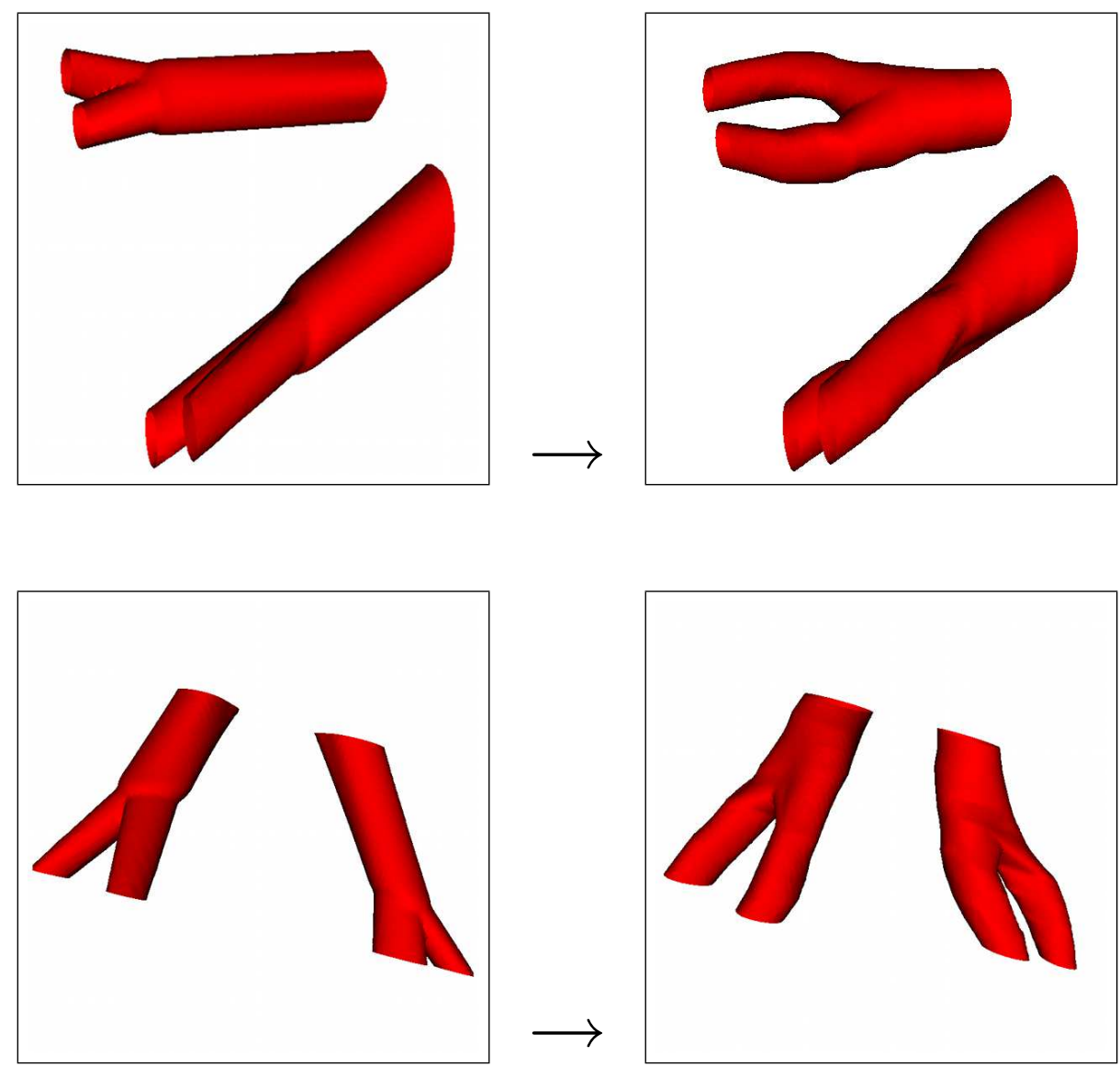

Figure 22: Two views of the reconstruction from two cuts on the left, same views of the full data (10 cuts) reconstruction on the right.

\section{Conclusions and perspectives}

In this work, we have designed a user code in order to extract blood vessels from imagery. These images are obtained from scanner and correspond to $2 D$ transversal cuts. To reconstruct the $3 D$ blood vessels, we can apply our approach with a limited number of transversal cuts (even two), where human intervention for segmentation is limited to the number of these cuts. To correct the positions of vessels and obtain more accurate results, we can include additional $2 D$ transversal cuts to enrich the first reconstruction, without human intervention. In the case where the full data are available, the construction strategy is different and human intervention will be only necessary on the first cut. The reconstructed geometry is defined by the zero level of a function defined for all grid size.

In a future work, knowing that the image processing of the full data are very costly when we use hundreds of images, we plan to compare the algorithm (5.2) proposed in section 5 with those of [19] or Chan-Vese [4] based on the Mumford-Shah algorithm. The Mumford-Shah algorithm [19], modified as suggested in section 5, can take into account the grey-levels and should be compared in terms of accuracy and cost with our method, on extreme situations. Nevertheless, note that our method has never failed on our real data. 
In addition, we expect to apply some human intervention to disconnect some arteries when they finish their course.

Note that the blood vessels, described by a level set function, are easily integrated in a fluid mechanic solver (Figure 23).

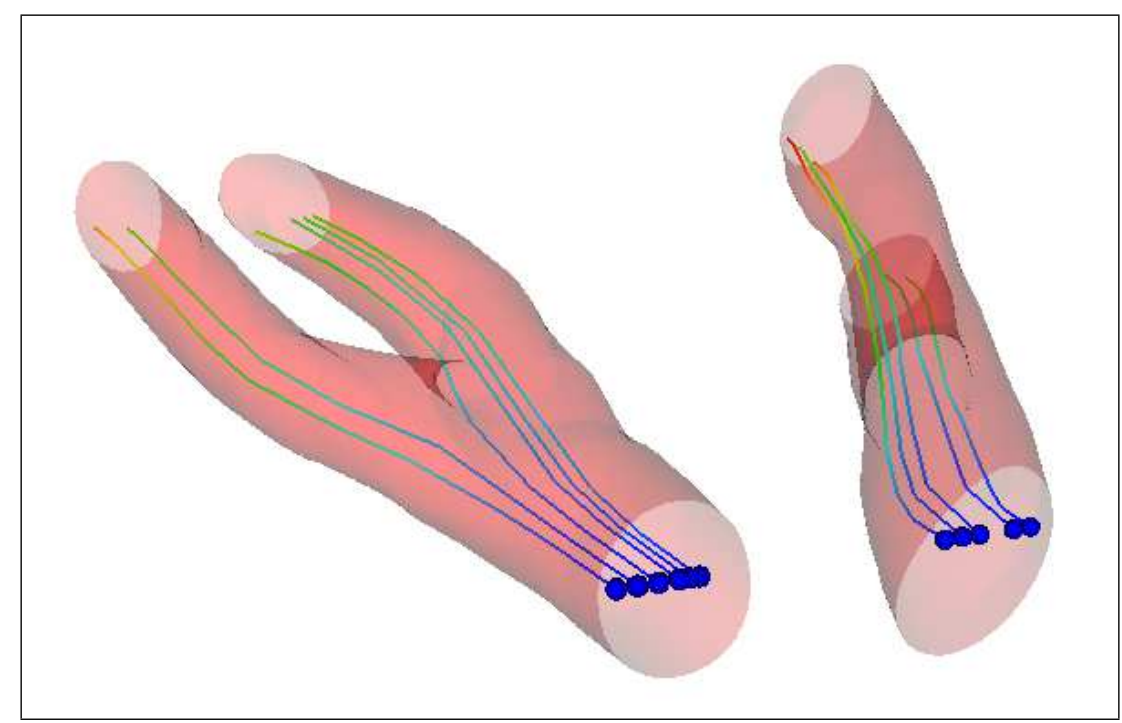

Figure 23: Flow, represented by streamlines, inside a $3 D$ reconstructed geometry.

For further developments, we plan to simulate blood flows modeled by non-Newtonian fluids in elastic vessels. The elastic models of geometries in fluid structure interactions are well developed in [5] with level set representation.

\section{References}

[1] Julie Benech. Spécificité de la mise en oeuvre de la tomographie dans le domaine de l'arc électrique-Validité en imagerie médicale. $\mathrm{PhD}$ thesis, Université de Toulouse, Université Toulouse III-Paul Sabatier, 2008.

[2] Marc Bernot. Transport optimal et irrigation. PhD thesis, École normale supérieure de CachanENS Cachan, 2005.

[3] M Bertero and P Boccacci. Introduction to inverse problems in imaging. 1998. Bristol, UK: IOP Publishing, 1985.

[4] Tony F Chan and Luminita A Vese. A level set algorithm for minimizing the mumford-shah functional in image processing. In Variational and Level Set Methods in Computer Vision, 2001. Proceedings. IEEE Workshop on, pages 161-168. IEEE, 2001. 
[5] Georges-Henri Cottet and Emmanuel Maitre. A level-set formulation of immersed boundary methods for fluid-structure interaction problems. Comptes Rendus Mathematique, 338(7):581$586,2004$.

[6] James L Evans, Kok-Hwee Ng, Stephen G Wiet, Michael J Vonesh, William B Burns, Martin G Radvany, Bonnie J Kane, Charles J Davidson, Sanford I Roth, Barry L Kramer, et al. Accurate three-dimensional reconstruction of intravascular ultrasound data spatially correct three-dimensional reconstructions. Circulation, 93(3):567-576, 1996.

[7] G Finet, E Maurincomme, A Tabib, RJ Crowley, I Magnin, R Roriz, J Beaune, and M Amiel. Artifacts in intravascular ultrasound imaging: analyses and implications. Ultrasound in medicine $\& 3$ biology, 19(7):533-547, 1993.

[8] Mireille Garreau, Jean Louis Coatrieux, Rene Collorec, and Christine Chardenon. A knowledgebased approach for 3-d reconstruction and labeling of vascular networks from biplane angiographic projections. Medical Imaging, IEEE Transactions on, 10(2):122-131, 1991.

[9] EN Gilbert. Minimum cost communication networks. Bell System Technical Journal, 46(9):2209-2227, 1967.

[10] P Jiménez Guerra and B Rodriguez-Salinas. A general solution of the monge-kantorovich mass-transfer problem. Journal of mathematical analysis and applications, 202(2):492-510, 1996.

[11] Sethian J.A. Level set methods and fast marching methods, 1999. ISBN 0-521-64557-3.

[12] Mélissa Jourdain. Reconstruction 3D des artères par imagerie intravasculaire ultrasonore (IVUS) et angiographie monoplan. Universite de Montreal, 2009.

[13] Michael Kass, Andrew Witkin, and Demetri Terzopoulos. Snakes: Active contour models. International journal of computer vision, 1(4):321-331, 1988.

[14] RO Kenet, EM Herrold, GJ Tearney, KK Wong, JP Hill, and JS Borer. 3-d quantitative assessment of coronary luminal morphology using biplane digital angiography. In Computers in Cardiology, 1988. Proceedings., pages 13-17. IEEE, 1988.

[15] Richard I Kitney, Lincoln Moura, and Keith Straughan. 3-d visualization of arterial structures using ultrasound and voxel modelling. In Intravascular ultrasound, pages 135-143. Springer, 1989.

[16] Eric Maurincomme, Isabelle E Magnin, Gerard Finet, and Robert Goutte. Methodology for three-dimensional reconstruction of intravascular ultrasound images. In Medical Imaging VI, pages 26-34. International Society for Optics and Photonics, 1992.

[17] John C Messenger, SY James Chen, John D Carroll, JEB Burchenal, Kathy Kioussopoulos, and Bertron M Groves. 3d coronary reconstruction from routine single-plane coronary angiograms: clinical validation and quantitative analysis of the right coronary artery in 100 patients. The International Journal of Cardiac Imaging, 16(6):413-427, 2000.

[18] Luciano Modica and Stefano Mortola. Un esempio di $\gamma$-convergenza. Boll. Un. Mat. Ital. B (5), 14(1):285-299, 1977. 
[19] David Mumford and Jayant Shah. Optimal approximations by piecewise smooth functions and associated variational problems. Communications on pure and applied mathematics, 42(5):577685, 1989.

[20] Cecil D Murray. The physiological principle of minimum work: I. the vascular system and the cost of blood volume. Proceedings of the National Academy of Sciences of the United States of America, 12(3):207, 1926.

[21] Edouard Oudet and Filippo Santambrogio. A modica-mortola approximation for branched transport and applications. Archive for rational mechanics and analysis, 201(1):115-142, 2011.

[22] N. Pal and S. Pal. A review on image segmentation techniques. Pattern Recognition, 26(9):1277$1294,1993$.

[23] Kenneth Rosenfield, Douglas W Losordo, K Ramaswamy, John O Pastore, RE Langevin, Syed Razvi, Bernard D Kosowsky, and Jeffrey M Isner. Three-dimensional reconstruction of human coronary and peripheral arteries from images recorded during two-dimensional intravascular ultrasound examination. Circulation, 84(5):1938-1956, 1991.

[24] M Senasli, L Garnero, A Herment, and E Mousseaux. Reconstruction 3d de vaisseaux à partir d'un faible nombre de projections à l'aide de contours déformables. In Colloque sur le traitement du signal et des images, FRA, 199716. GRETSI, Groupe dEtudes du Traitement du Signal et des Images, 1997.

[25] D. Sherknies. Atlas numérique spatio-temporel des artères coronaires. Université de Montréal, 2003.

[26] CJ Slager, JJ Wentzel, JA Oomen, JC Schuurbiers, R Krams, C Von Birgelen, A Tjon, PW Serruys, and PJ De Feyter. True reconstruction of vessel geometry from combined x-ray angiographic and intracoronary ultrasound data. In Seminars in interventional cardiology: SIIC, volume 2, pages 43-47, 1997.

[27] Cornelis J Slager, Jolanda J Wentzel, Johan CH Schuurbiers, Jan AF Oomen, Jeroen Kloet, Rob Krams, Clemens Von Birgelen, Willem J Van Der Giessen, Patrick W Serruys, and Pim J De Feyter. True 3-dimensional reconstruction of coronary arteries in patients by fusion of angiography and ivus (angus) and its quantitative validation. Circulation, 102(5):511-516, 2000 .

[28] F Sureda, I Bloch, C Pellot, and A Herment. Reconstruction 3d de vaisseaux sanguins par fusion de données à partir d'images angiographiques et échographiques. Traitement du Signal, 11(6):525-540, 1994.

[29] Alexandru Telea and Jarke J. van Wijk. An augmented fast marching method for computing skeletons and centerlines. In VISSYM '02: Proceedings of the symposium on Data Visualisation 2002, pages 251-ff, Aire-la-Ville, Switzerland, Switzerland, 2002. Eurographics Association.

[30] Harm ten Hoff, A Korbijn, Th H Smit, JFF Klinkhamer, and N Bom. Imaging artifacts in mechanically driven ultrasound catheters. In Intravascular ultrasound, pages 195-199. Springer, 1989 . 
[31] Luong Van Tran, Robert C Bahn, and Jack Sklansky. Reconstructing the cross sections of coronary arteries from biplane angiograms. Medical Imaging, IEEE Transactions on, 11(4):517529, 1992.

[32] Kateryna VORONETSKA, Guillaume VINAY, Anthony WACHS, and Jean-Paul CALTAGIRONE. Méthode level-set dans la modélisation des écoulements diphasiques. 20ème Congrès Français de Mécanique, 28 août/2 sept. 2011-25044 Besançon, France (FR), 2011.

[33] Steve Webb. The contribution, history, impact and future of physics in medicine. Acta Oncologica, 48(2):169-177, 2009.

[34] Qinglan Xia. Optimal paths related to transport problems. Communications in Contemporary Mathematics, 5(02):251-279, 2003. 